\title{
Correction to: Angiogenesis Genotyping and Clinical Outcomes in Patients with Advanced Hepatocellular Carcinoma Receiving Sorafenib: The ALICE-2 Study
}

\author{
Luca Faloppi ${ }^{1,2}$ - Marco Puzzoni ${ }^{2} \cdot$ Andrea Casadei Gardini $^{3} \cdot$ Nicola Silvestris $^{4}$ - Gianluca Masi ${ }^{5}$. Giorgia Marisi ${ }^{3}$. \\ Caterina Vivaldi ${ }^{5}$. Cosmo Damiano Gadaleta ${ }^{4}$. Pina Ziranu ${ }^{2}$. Maristella Bianconi ${ }^{6}$. Cristian Loretelli ${ }^{7}$. \\ Laura Demurtas $^{2}$ - Eleonora Lai ${ }^{2} \cdot$ Riccardo Giampieri $^{8} \cdot$ Eva Galizia $^{1} \cdot$ Paola Ulivi $^{9} \cdot$ Nicola Battelli $^{1} \cdot$ Alfredo Falcone $^{5}$. \\ Stefano Cascinu ${ }^{10} \cdot$ Mario Scartozzi $^{2,11}$
}

Published online: 10 November 2020

(c) Springer Nature Switzerland AG 2020

\section{Correction to: Targeted Oncology (2020) 15:115-126 https://doi.org/10.1007/s11523-020-00698-x}

Faloppi L, Puzzoni M, Casadei Gardini A, Silvestris N, Masi G, Marisi G, et al. Angiogenesis Genotyping and Clinical Outcomes in Patients with Advanced Hepatocellular Carcinoma Receiving Sorafenib: The ALICE-2 Study. Targeted Oncology. 2020;15:115-126

Page 115 and 126: The listing of the author names and affiliations, which previously read:

"Luca Faloppi 1,2, Marco Puzzoni², Andrea Casadei Gardini $^{3}$, Nicola Silvestris ${ }^{4}$, Gianluca Masi ${ }^{5}$, Giorgia Marisi $^{3}$, Caterina Vivaldi ${ }^{5}$, Cosmo Damiano Gadaleta ${ }^{4}$, Pina Ziranu ${ }^{2}$, Maristella Bianconi ${ }^{6}$, Cristian Loretelli ${ }^{7}$,

The original article can be found online at https://doi.org/10.1007/ s11523-020-00698-x.

Mario Scartozzi

marioscartozzi@gmail.com

1 Medical Oncology Unit, Macerata General Hospital, ASUR Marche AV3, Macerata, Italy

2 Medical Oncology Unit, University Hospital and University of Cagliari, Cagliari, Italy

3 Medical Oncology Unit, IRCSS-IRST, Meldola, Italy

4 Medical Oncology Unit, IRCCS Giovanni Paolo II Cancer Center, Bari, Italy

5 Medical Oncology Unit, University of Pisa, Pisa, Italy

6 Medical Oncology Unit, San Benedetto del Tronto Hospital, ASUR Marche AV5, San Benedetto del Tronto, Italy

7 International Center for T1D, Department of Biomedical and Clinical Sciences, Pediatric Clinical Research Center Romeo ed Enrica Invernizzi "L. Sacco”, University of Milan, Milan, Italy
Laura Demurtas ${ }^{2}$, Eleonora Lai $^{2}$, Riccardo Giampieri ${ }^{8}$, Eva Galizia $^{1}$, Paola Ulivi ${ }^{3}$, Nicola Battellii ${ }^{1}$, Alfredo Falcone ${ }^{5}$, Stefano Cascinu ${ }^{9}$, Mario Scartozzi ${ }^{2,10}$

${ }^{1}$ Medical Oncology Unit, Macerata General Hospital, ASUR Marche AV3, Macerata, Italy

${ }^{2}$ Medical Oncology Unit, University Hospital and University of Cagliari, Cagliari, Italy

${ }^{3}$ Medical Oncology Unit, IRCSS-IRST, Meldola, Italy

${ }^{4}$ Medical Oncology Unit, IRCCS Giovanni Paolo II Cancer Center, Bari, Italy

${ }^{5}$ Medical Oncology Unit, University of Pisa, Pisa, Italy

8 Oncology Department, Polytechnic University of Marche, Ancona, Italy

9 Istituto Scientifico Romagnolo per lo Studio e la Cura dei Tumori (IRST) IRCCS, Meldola, FC, Italy

10 Medical Oncology Unit, University of Modena, Modena, Italy

11 Oncologia Medica, Azienda Ospedaliera Universitaria Cagliari, Presidio Policlinico Universitario "Duilio Casula", Km 4500 Bivio per Sestu, S.S. 554, 09042 Monserrato, CA, Italy 
${ }^{6}$ Medical Oncology Unit, San Benedetto del Tronto Hospital, ASUR Marche AV5, San Benedetto del Tronto, Italy

${ }^{7}$ International Center for T1D, Pediatric Clinical Research Center Romeo ed Enrica Invernizzi “L. Sacco" Department of Biomedical and Clinical Sciences, University of Milan, Milan, Italy

${ }^{8}$ Oncology Department, Polytechnic University of Marche, Ancona, Italy

${ }^{9}$ Medical Oncology Unit, University of Modena, Modena, Italy

${ }^{10}$ Oncologia Medica, Azienda Ospedaliera Universitaria Cagliari, Presidio Policlinico Universitario "Duilio Casula", S.S. 554, Km 4,500 Bivio per Sestu, Monserrato, CA 09042, Italy"

should read:

"Luca Faloppi ${ }^{1,2}$, Marco Puzzoni ${ }^{2}$, Andrea Casadei Gardini ${ }^{3}$, Nicola Silvestris ${ }^{4}$, Gianluca Masi ${ }^{5}$, Giorgia Marisi ${ }^{3}$, Caterina Vivaldi $^{5}$, Cosmo Damiano Gadaleta ${ }^{4}$, Pina Ziranu ${ }^{2}$, Maristella Bianconi ${ }^{6}$, Cristian Loretelli ${ }^{7}$, Laura Demurtas ${ }^{2}$, Eleonora Lai $^{2}$, Riccardo Giampieri ${ }^{8}$, Eva Galizia ${ }^{1}$, Paola Ulivi ${ }^{9}$, Nicola Battelli ${ }^{1}$, Alfredo Falcone ${ }^{5}$, Stefano Cascinu ${ }^{10}$, Mario Scartozzi ${ }^{2,11}$

${ }^{1}$ Medical Oncology Unit, Macerata General Hospital, ASUR Marche AV3, Macerata, Italy
${ }^{2}$ Medical Oncology Unit, University Hospital and University of Cagliari, Cagliari, Italy

${ }^{3}$ Medical Oncology Unit, IRCSS-IRST, Meldola, Italy

${ }^{4}$ Medical Oncology Unit, IRCCS Giovanni Paolo II Cancer Center, Bari, Italy

${ }^{5}$ Medical Oncology Unit, University of Pisa, Pisa, Italy

${ }^{6}$ Medical Oncology Unit, San Benedetto del Tronto Hospital, ASUR Marche AV5, San Benedetto del Tronto, Italy

${ }^{7}$ International Center for T1D, Pediatric Clinical Research Center Romeo ed Enrica Invernizzi "L. Sacco" Department of Biomedical and Clinical Sciences, University of Milan, Milan, Italy

${ }^{8}$ Oncology Department, Polytechnic University of Marche, Ancona, Italy

${ }^{9}$ Istituto Scientifico Romagnolo per lo Studio e la Cura dei Tumori (IRST) IRCCS, Meldola (FC), Italy

${ }^{10}$ Medical Oncology Unit, University of Modena, Modena, Italy

${ }^{11}$ Oncologia Medica, Azienda Ospedaliera Universitaria Cagliari, Presidio Policlinico Universitario "Duilio Casula", S.S. 554, Km 4,500 Bivio per Sestu, Monserrato, CA 09042, Italy 